\title{
LOS MAYAS DEL CLÁSICO TARDÍO Y TERMINAL. UNA PROPUESTA ACERCA DE LA DINÁMICA DEMOGRÁFICA DE ALGUNOS GRUPOS MAYAS PREHISPÁNICOS: JAINA, PALENQUE Y COPÁN
}

\author{
LOURDES MÁRqueZ MoRfín \\ Escuela Nacional de Antropología e Historia \\ PATriCia Hernández Espinoza \\ Instituto Nacional de Antropología e Historia
}

\begin{abstract}
Resumen: En este trabajo analizamos la información demográfica obtenida en estudios específicos de algunos grupos mayas procedentes de asentamientos del Clásico y el Posclásico, de zonas costeras de la Península de Yucatán: Jaina, Xcaret y Chac Mool, y de otros sitios como Palenque y Copán. Presentamos un panorama general sobre la posible dinámica poblacional de estos grupos mayas, en particular datos acerca de la mortalidad, la fecundidad, la sobrevivencia y la esperanza de vida, mediante la metodología paleodemográfica. Integramos una muestra de 676 esqueletos de estos sitios, a los cuales se estimó sexo y edad, y obtuvimos su distribución por edades. Resalta el subregistro infantil (menores de un año) en varias series. En la evaluación específica de cada sitio, las series de Jaina y Copán presentan una adecuada composición por grupos de edad y porcentajes representativos de niños. La edad media a la muerte calculada es de 28.5 años. La mortalidad es similar para Palenque y Copán, y casi el doble para Jaina. La tasa global de fecundidad, que estima el número de hijos, es de 5.0 para Palenque y Copán, y de 8.8 para Jaina. Para este último grupo, la fecundidad es de 63.9, que es la tasa más alta obtenida. Los resultados son discutidos considerando cuestiones teóricas y metodológicas, así como los patrones demográficos específicos de cada serie, con relación a las tendencias de otros grupos antiguos.
\end{abstract}

Palabras clave: paleodemografía, mayas, fecundidad, mortalidad.

AвstRAct: In this paper we analyze demographic information obtained from specific studies on several Maya settlements from Classic and Postclassic periods, some from coastal Yucatan Peninsula: Jaina, Xcaret and Chac Mool, and other sites such as, Palenque and Copán. We present an overview of the possible population dynamic of these Mayan groups, including data about mortality, fertility, survival and life expectancy, by paleodemographic methodology. A sample of 676 skeletons was integrated from these sites. We estimated sex and age at death, and age distribution was obtained. Child (under one year) underrepresentation was observed in several series. Series from Jaina and Copán have an appropriate composition by age groups. The average age at death of 28.5 years was calculated. Mortality is similar to Palenque and Copán and almost double for Jaina. The total fertility rate, which estimates the number of children per women, is 5.0 for Palenque and Copán, and 8.8 to Jaina. For the latter group the fertility rate is 63.9 , which is the highest rate obtained. Results are discussed considering theoretical and methodological issues, as well as specific demographic patterns of each series, with respect to trends from other ancient groups.

KeYwoRDs: paleodemography, Maya, fertility, mortality.

RECEPCIÓN: 9 de octubre de 2012.

ACEPTACIÓN: 15 de marzo de 2013. 



\title{
LOS MAYAS DEL CLÁSICO TARDÍO Y TERMINAL. UNA PROPUESTA ACERCA DE LA DINÁMICA DEMOGRÁFICA DE ALGUNOS GRUPOS MAYAS PREHISPÁNICOS: JAINA, PALENQUE Y COPÁN
}

\author{
LouRdes MÁrqueZ Morfín \\ Escuela Nacional de Antropología e Historia \\ Patricia Hernández Espinoza \\ Instituto Nacional de Antropología e Historia
}

\section{Introducción}

Los estudios paleodemográficos en población maya prehispánica son difíciles de realizar debido a una diversidad de factores tales como prácticas funerarias diferenciales por sexo y edad; políticas de excavación de cada sitio; deterioro de los restos óseos, entre otros. La base de la paleodemografía radica en contar con una muestra poblacional de tamaño y composición por edad y sexo adecuada. Sin embargo, la realidad es que esta situación no es común, por lo que existen limitantes que problematizan obtener dinámicas demográficas generales para los grupos mayas antiguos con una evolución cultural compleja y heterogénea.

Con el objetivo de calcular los parámetros demográficos principales, conformamos una amplia muestra esquelética maya, en la cual integramos los resultados de la estimación osteológica de edad para varios sitios (Jaina, Xcaret, Chac Mool y Palenque, en México, y Copán, en Honduras) (figura 1). La muestra quedó compuesta por 676 individuos, de los periodos Clásico Tardío-Terminal y Posclásico, y de ella se calcularon tendencias generales sobre la dinámica demográfica. Utilizamos técnicas estandarizadas, tanto para estimar la edad, como para los parámetros demográficos.

\section{La población maya del Clásico Tardío y Terminal}

El Clásico Tardío (600 a 800 d.C.) se caracteriza por la expansión territorial, la complejidad social y el aumento en la densidad de población (Sharer, 1999), lo que constituye uno de los factores principales que permiten plantear interrogantes 


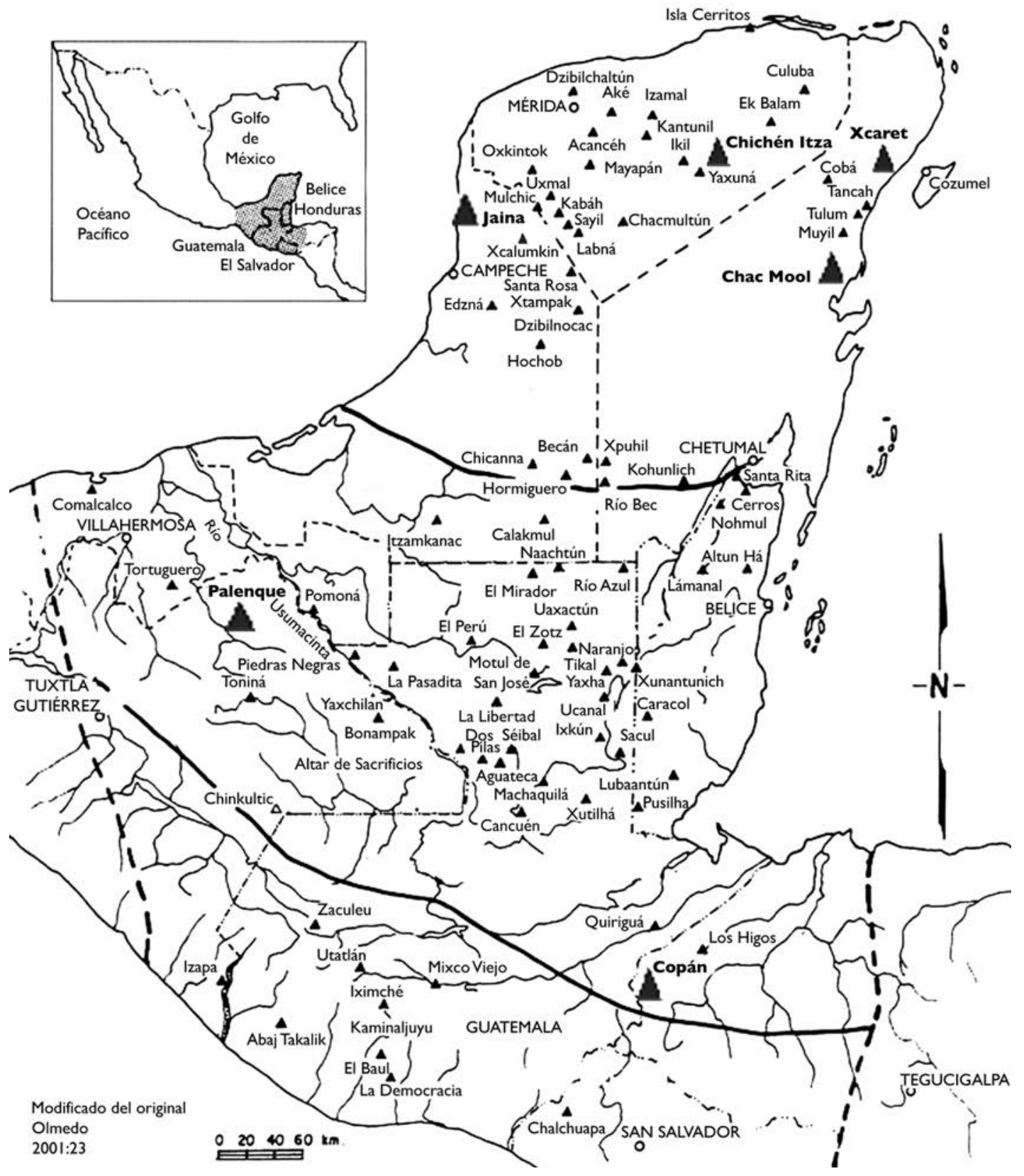

FIGURA 1. Localización de los sitios mencionados en el texto. 
sobre la calidad y condiciones de vida de estos individuos, así como de su longevidad, su adaptación al medio ambiente físico y social, y sus niveles de sobrevivencia y de reproducción.

La alta densidad de población de los grandes centros urbanos conlleva a un deterioro en la salud, debido principalmente a infecciones provocadas por el hacinamiento, así como a la contaminación de agua y alimentos por basura y desechos orgánicos (Cohen y Crane-Kramer, 2007; Márquez Morfín y Hernández Espinoza, 2004, 2006a: 97-99 y 2008). De acuerdo con Sharer (1999: 345), el crecimiento demográfico en el Clásico intensificó las presiones sobre un sistema de subsistencia cada vez más complejo y vulnerable, precisamente mientras aumentaba la desnutrición y las enfermedades y se reducía la productividad. Los estudios demográficos sobre la población maya señalan que, pese a los altos índices de mortalidad, tanto a nivel infantil como general, la fecundidad era lo suficientemente alta para mantener el crecimiento positivo en estos grupos (Hernández Espinoza y Márquez Morfín, 2006a, 2006b y 2007; Storey, 1992a, 1992b y 1997).

Durante el siglo ix, periodo conocido como Clásico Terminal (790 a 889 d.C.) en la mayor parte de los centros mayas de las Tierras Bajas meridionales y centrales, se produce el abandono de ciudades importantes como Copán y Tikal. En Palenque hay registros de un descenso considerable de población, así como de un deterioro en la salud de los integrantes de la elite secundaria (Haviland, 2003; Liendo Stuardo, 2004; Márquez et al., 2002; Sharer, 1999: 333; Storey, 1997 y 1999).

En la Península de Yucatán, hacia el mismo periodo, Chichén Itzá se transformó en un poderoso centro regional que controló a los asentamientos menores que se localizaban en su periferia, asegurando el área comprendida entre la costa norte y la región central de la península (Cobos Palma, 2004: 538), estableciendo e interviniendo sus propios puertos de embarque y expandiendo su influencia a lo largo de la costa oriental y occidental de la Península de Yucatán (Andrews et al., 1988; Andrews y Robles, 1985; Robles y Andrews, 1986; Cobos, 2004: 538-539).

Hacia esa misma época, las crónicas mayas mencionan la llegada a tierras yucatecas de un grupo putún conocido como itzaes, guerreros y comerciantes procedentes de las Tierras Bajas del Golfo de México, que lograron dominar el comercio costero en torno a la Península de Yucatán, de tal manera que hacia el Posclásico Temprano controlaban los principales puertos marítimos de la costa de Quintana Roo, entre ellos el de Chac Mool (Sharer, 1999: 338).

A la caída de Chichén Itzá, los itzaes migraron hacia distintos puntos de la costa oriental, que abandonaron con la caída de Mayapán, alrededor del año 1441 d.C., de tal manera que al momento de la llegada de los españoles algunos sitios que antes fueron importantes enclaves comerciales habían perdido su importancia y se convirtieron en asentamientos más o menos poblados, cuyo patrón de subsistencia estaba basado en la explotación de recursos marinos, como es el caso de Chac Mool y de la antigua Polé, después conocida como Xcaret (Chacín, 2003; González Licón y Terrones González, 2003 y 2004). 
Esta secuencia de acontecimientos sugiere también sucesos que afectaron la forma de vida de los pobladores de esta región y permite planear varias interrogantes: ¿Cuáles fueron las consecuencias para la duración de la vida de estos individuos? ¿Los diferentes contextos ecológicos tuvieron alguna repercusión en el crecimiento demográfico de cada sitio? ¿Se restringieron los límites de sobrevivencia? ¿Cómo se vieron afectados los niveles de mortalidad? Éstas son las preguntas que guían este trabajo y que trataremos de ir analizando a partir de los vestigios humanos de los pobladores de Jaina, Xcaret, Chac Mool y Palenque, en México, y Copán, en Honduras, de los cuales contamos con información valiosa e interesante para entender su dinámica demográfica.

\section{Contexto ecológico y cultural de las series estudiadas. Las grandes ciudades}

\section{Palenque, Chiapas}

La serie osteológica está fechada para el Clásico Tardío-Terminal (650-800 d.C). Los enterramientos de esta serie proceden de estructuras de edificios con distintas funciones: rituales, administrativas, de control político y habitacionales, como el Templo XV, el de la Cruz y el de la Cruz Foliada, los cuales están ligados con linajes de los gobernantes de Palenque.

Los individuos que vivieron en las unidades habitacionales de los grupos $\mathrm{C}, \mathrm{B}$, I, II y IV ${ }^{1}$ tenían una composición socioeconómica heterogénea. Estos edificios tuvieron funciones asociadas a actividades tanto domésticas como de carácter ritual y estaban organizados alrededor de plazas en grupos que formaban unidades mayores a manera de barrios. Los grupos residenciales, de acuerdo con la información etnográfica, podrían estar conformados por familias extensas y por individuos no emparentados, cohesionados por un individuo "de mayor prestigio por su cercanía (en términos de parentesco) al fundador del grupo residencial” (Liendo Stuardo, 2000). En las unidades domésticas se llevaban a cabo las principales actividades de reproducción económica y social. A pesar de que, desde el punto de vista arquitectónico, son bastante similares, al interior se aprecian variaciones de tipo funcional y económico (Liendo Stuardo, 2004; López Bravo, 1995). El análisis de indicadores de salud de esta serie, como la hipoplasia del esmalte, la espongio hiperostosis, la criba orbitaria y las reacciones periósticas, apuntan a una alta frecuencia de anemia y procesos infecciosos, interpretados como infecciones gastrointestinales producto de parasitosis crónicas, que no permiten la correcta absorción de los nutrientes ingeridos. Los indicadores demográficos muestran una alta mortalidad en los primeros años de vida, tanto entre recién nacidos como entre menores de cinco años (Márquez Morfín y Hernández Espinoza, 2004 y 2006b).

\footnotetext{
${ }^{1}$ Los materiales óseos fueron excavados y proporcionados por el arqueólogo Arnoldo González. Esta serie está integrada por 172 individuos, a los que se adicionaron 51 individuos más que fueron excavados del Grupo IV por Robert y Bárbara Rands en la década de 1950 y analizados por Fascio Guillén (2003). Los esqueletos fueron analizados por las autoras.
} 
Durante el periodo Clásico, Copán fue la capital de una poderosa entidad que dominó la región maya sudoriental en la frontera con América Central. Situado en un fértil valle a 700 metros sobre el nivel del mar, tiene una larga estación lluviosa (mayo a enero) que permitió el desarrollo de un sistema de cultivo intensivo para alimentar a su población. Los estudios realizados por Webster et al. (2000) sugieren que hacia el 750 d.C. la densidad demográfica había aumentado hasta llegar a los 27750 habitantes. De las diversas excavaciones en este sitio se ha recuperado una extensa muestra de esqueletos (239 individuos), que proceden de los entierros localizados en el complejo 9N-8, denominado La Casa de los Bacabes, ubicado en el barrio residencial de Sepulturas de esta ciudad, correspondiente al momento de máxima densidad demográfica y de florecimiento de esta sociedad, en la transición del Clásico Tardío al Clásico Terminal (700 a 1000 d.C.) (Storey, 1997: 116). Copán, al igual que otros sitios del periodo Clásico del área maya, disminuyó rápidamente su población después del florecimiento del Clásico Tardío. Hacia el año 1200 d.C., el valle estaba completamente abandonado (Webster et al., 2000).

De este sitio maya, Storey analizó dos series denominadas Copán Urbano y Copán Rural (1992b, 1997 y 1999). Los estudios bioarqueológicos realizados en esta serie toman en consideración indicadores arqueológicos que muestran a esta sociedad como altamente estratificada. Respecto a los datos biosociales, se han presentado resultados de alta mortalidad en la niñez (menores de cinco años) así como entre los adolescentes y las mujeres jóvenes, como una característica de las poblaciones prehispánicas (Storey, 1992b, 1997 y 1999; Whittington, 1989). El análisis de indicadores de salud, como la hipoplasia del esmalte, la criba orbitaria, la espongio hiperostosis y las reacciones periósticas, ${ }^{2}$ revelan que el grupo tenía problemas de salud pública, reflejados en una alta frecuencia de procesos infecciosos, tanto entre la elite como entre los comunes, muy similares a los de otros grupos de su tiempo como los de Palenque (Márquez, et al. 2001; Márquez Morfín y Hernández Espinoza, 2008; Márquez Morfín et al., 2002; Storey et al., 2002; Whittington, 1989, 1991, 1992 y 1999; Wright, 1997).

\section{Los asentamientos costeros de la Península de Yucatán}

La isla de Jaina, Campeche

Situada frente a las costas de Campeche, en una zona de pantanos y manglares, Jaina es un asentamiento maya cuyo periodo de ocupación data entre los años 300 y 1000 d.C. La gran cantidad de evidencias arqueológicas, así como de enterramientos humanos, sugieren un periodo de ocupación continua por una comu-

\footnotetext{
${ }^{2}$ Estos indicadores (algunos específicos y otros generales) de lesiones en el esqueleto son utilizados como fuentes excelentes de información para evaluar la salud. Para mayor información véase a Goodman y Martin (2002).
} 
nidad integrada por familias de pescadores y gente encargada de las actividades administrativas, comerciales y religiosas del lugar (López Alonso y Serrano Sánchez, 1997). La serie fue recuperada durante las excavaciones realizadas en la temporada de campo de 1973-1974 (López Alonso y Serrano Sánchez, 1984) y está integrada por 105 individuos (cuadro 2). De la población representada en las múltiples figurillas encontradas en el sitio sabemos poco (Benavides, 2007). ${ }^{3}$ Los trabajos realizados en esta serie sugieren una alta mortalidad infantil y problemas de salud comunes a otras poblaciones mayas, como los procesos infecciosos (Márquez Morfín y Hernández Espinoza, 2007; Peña Reyes et al., 2007).

Chac Mool, Quintana Roo

De este sitio existen dos series osteológicas, ${ }^{4}$ una de ellas corresponde al Clásico Terminal y otra al Posclásico (González Licón y Cobos Palma, 2006). Chac Mool está situado en Punta Pájaros, entre las Bahías de la Ascensión y del Espíritu Santo. Hacia el Posclásico Temprano, su ubicación estratégica lo convirtió en un enclave comercial itzae, un punto intermedio en la ruta que seguían los mercaderes de la sal (Stuart y Stuart, 1993). Durante el dominio de Chichen Itzá, este asentamiento tenía las características de cualquier puerto actual, con una gran proporción de hombres y un crecimiento demográfico lento (Giannisis, 2006; Hernández Espinoza y Márquez Morfín, 2006b). El análisis de las costumbres funerarias y de los indicadores de estratificación social señalan una distribución más o menos igualitaria de la riqueza, donde las mujeres tenían el mismo estatus que los hombres, y en algunos casos mayor (Núñez Enríquez, 2003). Los indicadores de salud y nutrición marcan pocos problemas de salud y una alimentación basada en productos procesados derivados del maíz y en el consumo de proteína de origen animal (Márquez Morfín y Hernández Espinoza, 2006b).

Bajo el dominio de Mayapán, Chac Mool se consolidó como un enclave comercial de la costa; los flujos migratorios eran continuos, aunque el asentamiento había crecido también por un aumento en los niveles de fecundidad, que le permitió alcanzar una densidad demográfica moderada. A la caída de Mayapán (1441 d.C.), durante el Posclásico Tardío, Chac Mool perdió la importancia que tenía como puerto comercial, convirtiéndose en un asentamiento más de la región. Cambios importantes en el patrón funerario señalan que las diferencias sociales se habían profundizado, la riqueza se concentró en unos cuantos y la salud sufrió un deterioro importante. La dieta se basó en la explotación de recursos marinos y la población aumentó por un incremento principalmente en los niveles de fecundidad (Hernández Espinoza y Márquez Morfín, 2006b).

\footnotetext{
${ }^{3}$ La serie fue analizada por Lourdes Márquez y Rebeca Storey.

${ }^{4}$ Series analizadas por Lourdes Márquez y Patricia Hernández.
} 
Xcaret, Quintana Roo

El sitio de Xcaret se localiza en la costa oriental de la Península de Yucatán, en el actual estado de Quintana Roo, frente a la isla de Cozumel. Se le asocia con un asentamiento prehispánico conocido como Polé, un puerto antiguo que estuvo en uso hasta 1668 (Chacín, 2003). Polé es el primer pueblo citado en la leyenda de migración de Chumayel referente a los itzaes, y su nombre se deriva de los términos mayas P'o, que se refiere a la actividad comercial, y le, que significa "trampa" o "lazo" (Con, 1991). ${ }^{5}$

\section{Metodología}

Cada esqueleto fue analizado para estimar el sexo, a partir de la evaluación del dimorfismo sexual presente en el cráneo, la mandíbula y la pelvis (Bass, 1995; Buikstra y Ubelaker, 1994). Para estimar la edad en esqueletos se consideraron los diferentes indicadores, dependiendo del estado de conservación del material óseo, como cambios degenerativos en la superficie auricular del ilíaco, en la sínfisis del pubis y en el extremo de la cuarta costilla, o el patrón de desgaste dental, entre otros (Buikstra y Ubelaker, 1994; Lovejoy, 1985; Lovejoy et al., 1985). En los esqueletos subadultos (menores de 15 años) se estimó la edad a partir de los procesos de desarrollo y maduración dental (Ubelaker, 1989), de la calcificación de raíces y coronas, siguiendo la propuesta de Peña Reyes (Hernández Espinoza y Peña Reyes, 2010), y del grado de fusión de las epífisis.

\section{La muestra integral}

La muestra quedó formada por 676 esqueletos de las colecciones esqueléticas de Palenque, Copán, Jaina, Xcaret y Chac Mool, cuyo rango de edad va desde recién nacidos hasta adultos de 70 años o más. La edad es la variable clave para los estudios demográficos. El primer indicador construido es la distribución de frecuencia por edad, y con base en estos cálculos se obtiene la media de edad de toda la población, que fue estimada en 28.5 años, cálculo muy próximo a la esperanza de vida de estos grupos, y una desviación estándar de 20.4; la mayoría de los individuos se encuentran entre los ocho y los 48 años, lo que da una gran rango de variación entre las edades, pero es lo esperado para una muestra que procede de distintos contextos arqueológicos.

Distribución de edades en subadultos (menores de 15 años)

La distribución por edad de los esqueletos es el punto de partida del análisis demográfico (cuadro 1). Se describe primero la frecuencia en los menores de 15 años,

\footnotetext{
${ }^{5}$ Las excavaciones efectuadas desde 1986 hasta 1994, por María José Con, dieron como resultado dos series osteológicas, una prehispánica y otra de la época del contacto, que fueron analizadas por Lourdes Márquez y Rebeca Storey. Los resultados de los estudios antropofísicos realizados en la serie prehispánica señalan problemas de salud semejantes a los de poblaciones mayas, sobre todo relacionados con procesos infecciosos (Márquez Morfín et al., 2002).
} 
en especial en el primer año de vida, dada su relevancia en la interpretación demográfica. El grupo de recién nacidos hasta los 11 meses de edad expresa tanto los efectos de la fecundidad como de la mortalidad infantil, ya que este indicador tiene repercusiones directas en la esperanza promedio de vida al nacimiento; el grupo siguiente, de uno a cuatro años, permite analizar la mortalidad de los primeros años de la niñez. A partir del grupo de cinco a nueve años, el análisis se efectúa por grupos quinquenales, para hacer comparables los datos con otros estudios similares. Los resultados en subadultos, si los contrastamos con la curva de mortalidad de las poblaciones antiguas (Livi-Bacci, 2002), donde se esperan porcentajes mayores para esta etapa de la vida, resaltan la reducida presencia de recién nacidos y de un año de edad en la serie maya, que significa un faltante del $22 \%$ de menores de un año (gráfica 1). El dato es explicable a partir de varias circunstancias: las prácticas funerarias diferenciales de acuerdo a los roles de género y edad, al deterioro diferencial de los restos, a la excavación parcial del sitio (Márquez Morfín y González Licón, 2010) o a la recuperación no sistemática de restos de neonato y de no-natos (Malgosa, 2010).

\begin{tabular}{|c|c|c|c|}
\hline Grupos de edad & Frecuancias & Porcentajes & $\begin{array}{l}\text { Porcentajes } \\
\text { acumulados }\end{array}$ \\
\hline 0 & 55 & 8.08 & 8.08 \\
\hline $1-4$ & 125 & 18.4 & 26.48 \\
\hline $5-9$ & 43 & 6.31 & 32.79 \\
\hline $10-14$ & 16 & 2.35 & 35.14 \\
\hline $15-19$ & 25 & 3.67 & 38.81 \\
\hline $20-24$ & 40 & 5.87 & 44.68 \\
\hline $25-29$ & 37 & 5.43 & 50.11 \\
\hline $30-34$ & 51 & 7.49 & 57.6 \\
\hline $35-39$ & 82 & 12.0 & 69.6 \\
\hline $40-44$ & 71 & 10.4 & 80.0 \\
\hline $45-49$ & 55 & 8.1 & 88.1 \\
\hline $50-55$ & 29 & 4.3 & 92.4 \\
\hline $55-59$ & 13 & 1.90 & 94.3 \\
\hline $60-64$ & 18 & 2.60 & 96.9 \\
\hline $65-69$ & 13 & 1.9 & 98.8 \\
\hline $70-74$ & 8 & 1.20 & 100.0 \\
\hline Totales & 676 & 100.0 & \\
\hline
\end{tabular}

Cuadro 1. Distribución de toda la serie por grupos de edad mixtos. Fuente: cálculos propios a partir del análisis osteológico de las series. 
En los sitios prehispánicos cuya práctica funeraria permitió la preservación de los esqueletos pequeños, como en el caso del barrio de Tlajinga 33, en Teotihuacan, el porcentaje de infantes es grande (Márquez et al., 2002: 318). En general, en las poblaciones antiguas los grupos de edad entre cinco y 14 años constituyen edades donde la mortalidad disminuye, pues una vez superados los embates de las enfermedades infantiles, la probabilidad de sobrevivencia es mayor; sin embargo, el número de individuos observado en la muestra integral, como ya mencionamos, está por debajo de lo esperado al compararlo con la curva de mortalidad antigua, donde las edades de mayor riesgo a morir se registran al nacimiento y antes de cumplir el primer año de vida, después el riesgo baja paulatinamente hasta los 10-14 años, desde donde se inicia el ascenso, asociado a los problemas que implicaba la entrada a la edad productiva y reproductiva (Acsádi y Neméskéri, 1970) (gráfica 1).

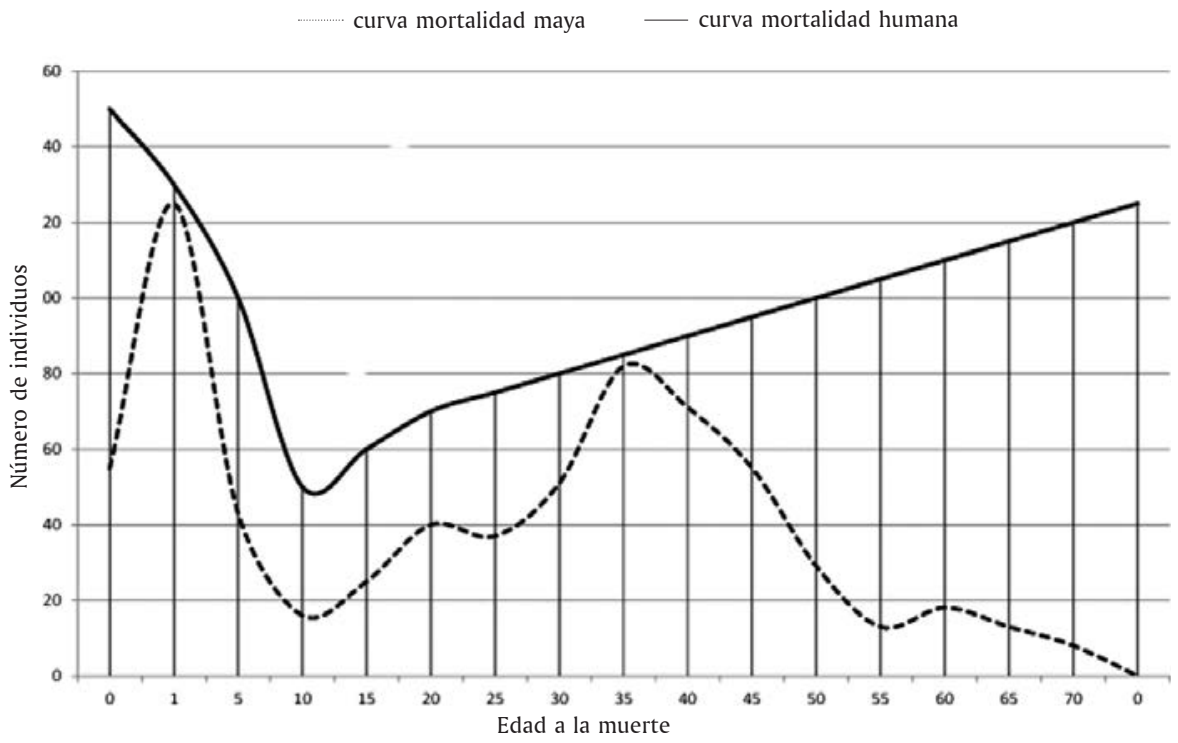

GRáfICA 1. Curva de mortalidad de la muestra maya vs. curva de mortalidad humana.

Para Jaina, el porcentaje en los menores de 15 años alcanza un 56\%; tan sólo el porcentaje de niños neonatos muertos alcanza el 19\%, y de un año, el $29 \%$, lo que significa una alta mortalidad infantil (gráfica 2).

Distribución de edades en adultos

La distribución por edad en adultos refleja una mayor mortalidad entre la cuarta y quinta décadas de vida, que, de acuerdo con Sauvine-Dugerdil (2006), se ha 
llamado la duración de la vida, o el número máximo que cada generación alcanza a vivir y que es acumulativo entre sociedades con iguales condiciones y estilos de vida y de adultos mayores de 40 años. ${ }^{6}$ La serie maya en la gráfica 1 tiene un pico alrededor de los 20 años y otro a los 35 .

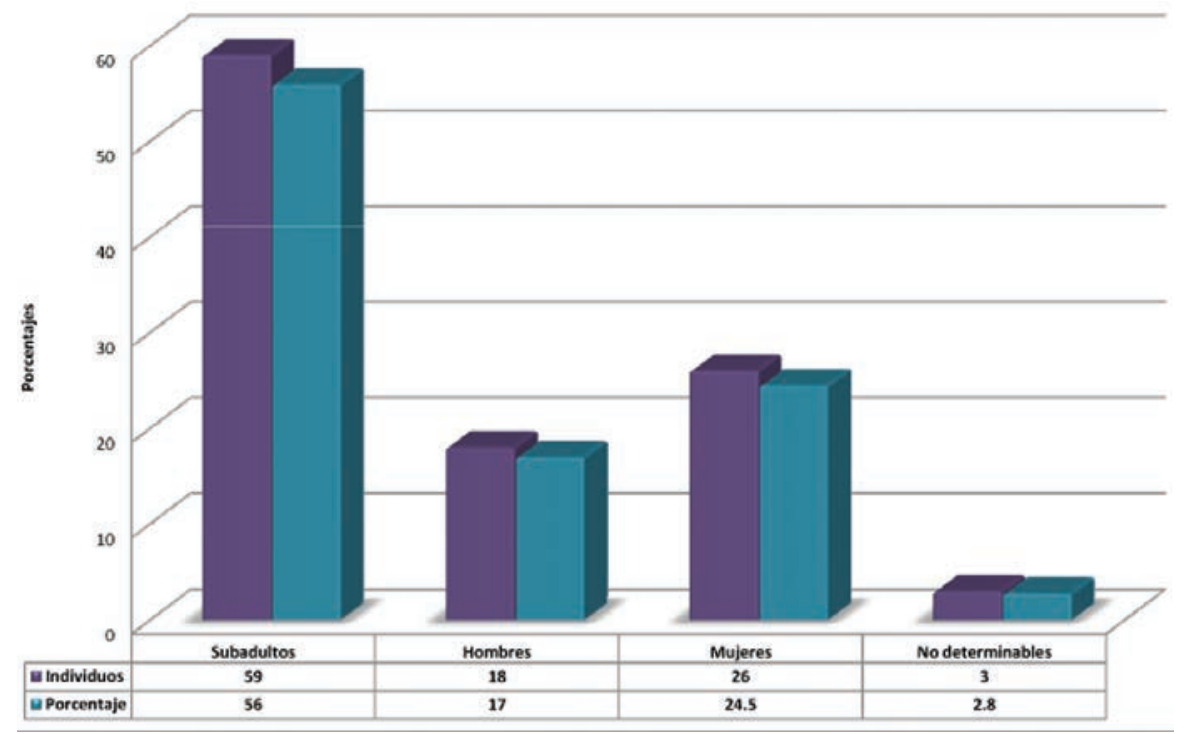

GráfICA 2. Serie de Jaina, Campeche. Distribución por sexo y edad.

\section{Análisis específico de cada serie}

Las disparidades en la distribución por edad se tratan de explicar a partir del contexto arqueológico y de las condiciones de recuperación de los materiales. Para ello realizamos un ejercicio estadístico con el fin de verificar la consistencia interna de la variable edad. Desagregamos la muestra por sitio, y se distribuyeron las edades individuales en cuatro categorías de edad (con intervalos de 15 años cada uno, con excepción del último, que es abierto). El propósito es estandarizar las diferencias existentes al agrupar en rangos más cortos, así tenemos las categorías de subadultos (0 a 14 años), adultos jóvenes (15 a 29 años), adultos medios (30 a 50 años) y adultos avanzados (mayores de 50 años). Los resultados se muestran en el cuadro 2 .

${ }^{6}$ Observación realizada hace dos décadas por Bocquet-Appel y Masset (1982), que cuestionaron la viabilidad de la paleodemografía. En otros trabajos hemos argumentado la ausencia de dichos individuos y existen propuestas para ajustar los faltantes, siempre y cuando tengamos la información arqueológica de la serie a estudiar. 


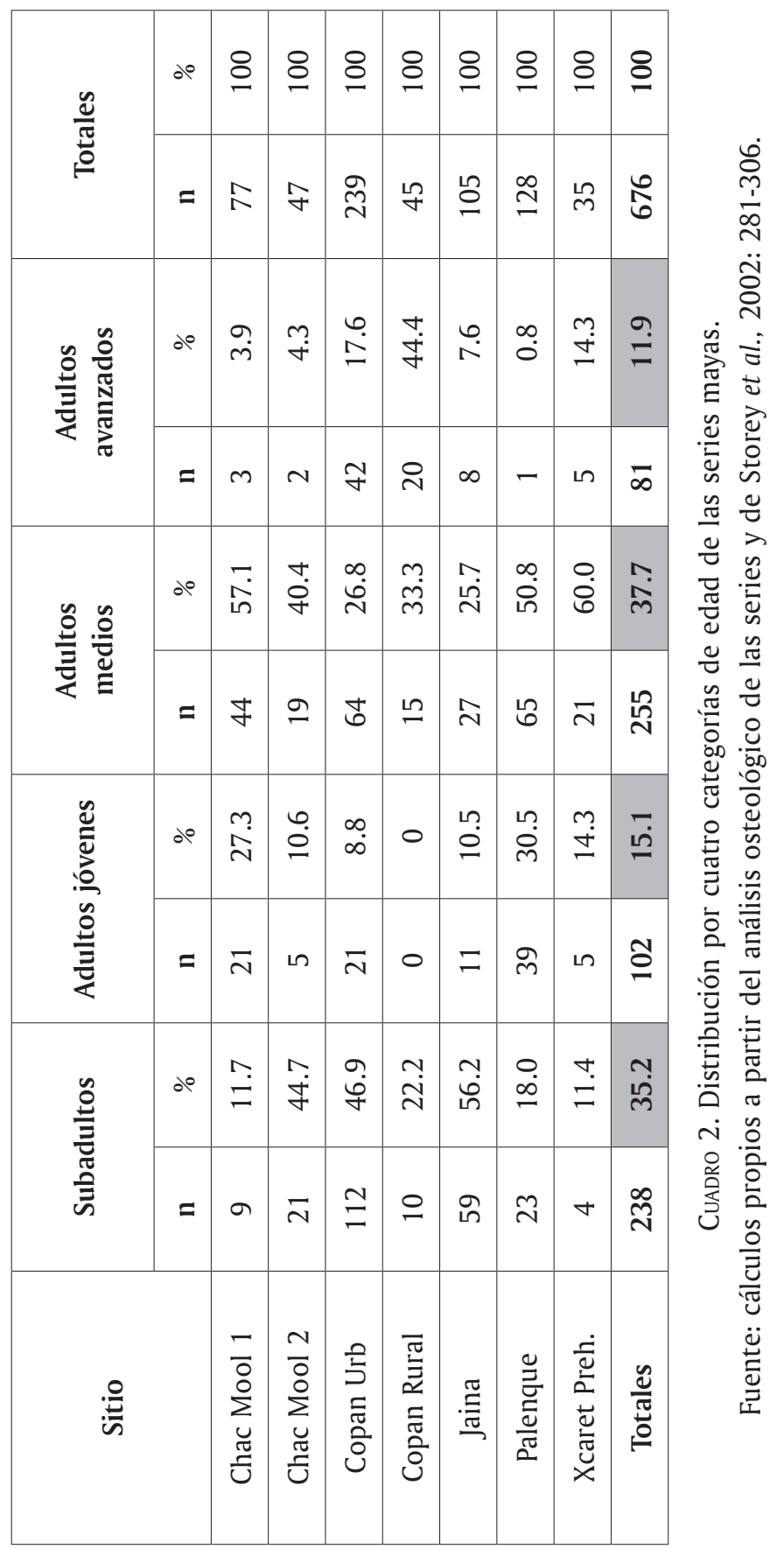


Las series con mayor cantidad de individuos "subadultos" son la de Copán Urbano $^{7}$ y Jaina, donde la excavación de esqueletos de neonatos e infantes permitió una adecuada recuperación, gracias a las prácticas funerarias en ollas, donde se preservaron los pequeños restos. De ahí que para el análisis demográfico se utilizan las series esqueléticas más completas. El resto de las colecciones de la muestra integral maya tienen una obvia subrepresentación de niños.

La siguiente categoría, "adultos jóvenes", también tiene subregistro, a excepción de Palenque, lo que podría interpretarse como que la mortalidad entre los individuos de estas edades no era muy alta, de ahí la poca representación. No obstante, habría que volver al registro arqueológico y a la historia social de estas poblaciones para llegar a tal conclusión. En esta categoría presentan subregistro las series de Chac Mool 2, Copán Rural y Xcaret prehispánico.

La mayoría de las series tienen altas concentraciones de "adultos medios", lo que indica la edad promedio de sobrevivencia de estos individuos. Hay que señalar que las mismas tres series que mencionamos para la categoría anterior tampoco tienen en este rango una buena representación. Los "adultos avanzados" o mayores de 50 años tienen escasa representación entre casi todas las series, con excepción de las dos series de Copán, lo que quizás tenga relación con la metodología utilizada para estimar la edad a la muerte, así como el error "interobservador", que es muy frecuente al aplicar metodologías basadas en la observación morfoscópica (gráfica 3).

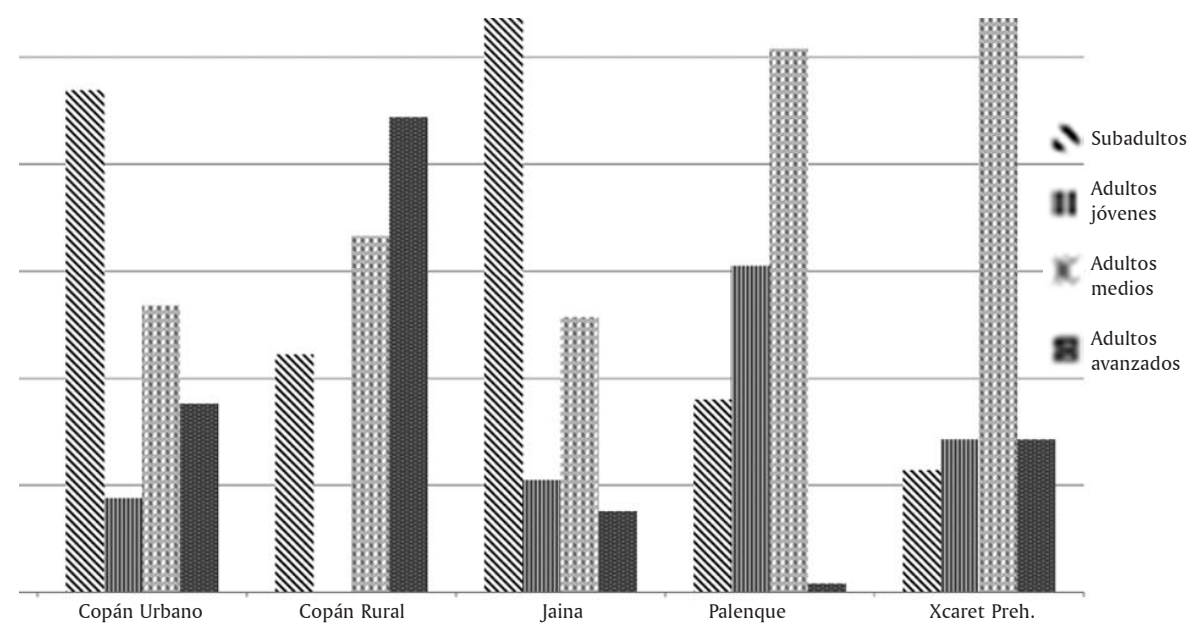

GRÁFICA 3. Distribución de edades a la muerte agrupadas en cuatro categorías por sitio.

${ }^{7}$ La recuperación del material óseo la llevó a cabo Rebeca Storey. Sus preguntas de investigación se enfocaron en conocer las condiciones y del estilo de vida de la población, para lo cual una de las variables imprescindibles para este tipo de análisis es el perfil demográfico, donde el número de esqueletos de recién nacidos y de infantes es vital. 
De acuerdo con los resultados de este análisis decidimos excluir las dos series de Chac Mool, la de Xcaret y la de Copán Rural (que constituían el 30\% de la muestra integral), ya que por los subregistros en determinadas edades las consideramos no aptas para llevar a cabo un estudio paleodemográfico. Analizamos de manera individual las series de Copán, Palenque y Jaina, cuya distribución y composición contiene las premisas para aplicar la metodología paleodemográfica. Consideramos que este tipo de ejercicio es indispensable para obtener resultados confiables, pues muchas veces se cree que agregando datos de alguna variable, para hacer las muestras más amplias, se tendrán mejores resultados, pero lo que se logra es la imposibilidad de interpretar los datos desde un enfoque biocultural.

\section{Evaluación de Jaina, Copán y Palenque}

La segunda parte del análisis, como mencionamos, consistió en estudiar las series de Jaina, Copán y Palenque desagregadas, lo que permite entender no sólo la consistencia de los datos, sino también la conformación de la serie de acuerdo con el contexto arqueológico y su viabilidad para el trabajo paleodemográfico.

\section{Análisis de subadultos}

Los individuos menores de cinco años representan el $48 \%$ de la serie de Jaina y el 39.8\% en Copán, pues hay más individuos representados en los dos primeros grupos de edad, mientras que en Palenque constituyen tan sólo el 7.0\%, indicando que estadísticamente la serie no tiene el número esperado de individuos recién nacidos (cuadro 3).

En la Isla de Jaina, de acuerdo con López Alonso y Serrano Sánchez (2007), el registro arqueológico revela que el sitio fue ocupado en numerosas ocasiones, de ahí la alta densidad de entierros. Al reiniciarse el estudio sistemático del sitio en la década de los setenta del siglo xx, se encontró que la mayoría de los entierros excavados corresponden a niños, por lo que la serie de ese lugar tiene una alta proporción de individuos menores de 15 años; en cambio, la serie de Palenque está sesgada, producto de la excavación de las criptas familiares, con una mayoría de adultos, quizá porque los niños, de acuerdo a su rango social, no eran sepultados ahí; ${ }^{8}$ en el caso de Copán, la exploración sistemática del sitio logró la recuperación cuidadosa de los restos óseos, consiguiendo así una buena representación de individuos en la mayoría de los grupos de edad. En la serie de Copán, el 50\% de los individuos tiene menos de 20 años de edad, reflejo de una población joven; en Jaina, un 50\% tiene menos de 10 años de edad, como consecuencia del gran número de infantes, y en la serie de Palenque, el $50 \%$ tiene menos de 30 años a la muerte. En este último caso, este porcentaje significa que la serie está integrada en su mayoría por individuos adultos.

\footnotetext{
${ }^{8}$ Para mayor información consultar a López Bravo, 1995 y 2000, y a Márquez y Hernández, 2004.
} 


\begin{tabular}{|c|c|c|c|c|c|c|}
\hline \multirow{2}{*}{$\begin{array}{l}\text { Grupos } \\
\text { de edad }\end{array}$} & \multicolumn{2}{|c|}{ Jaina } & \multicolumn{2}{|c|}{ Copán } & \multicolumn{2}{|c|}{ Palenque } \\
\hline & $\%$ & $\begin{array}{l}\text { Porcentaje } \\
\text { acumulado }\end{array}$ & $\%$ & $\begin{array}{l}\text { Porcentaje } \\
\text { acumulado }\end{array}$ & $\%$ & $\begin{array}{l}\text { Porcentaje } \\
\text { acumulado }\end{array}$ \\
\hline 0 & 18.9 & 18.9 & 15.1 & 15.1 & 0.0 & 0.0 \\
\hline 1 & 29.2 & 48.1 & 24.7 & 39.8 & 7.5 & 7.5 \\
\hline 5 & 5.7 & 53.8 & 5.0 & 44.8 & 8.0 & 15.5 \\
\hline 10 & 1.9 & 55.7 & 2.1 & 46.9 & 2.1 & 17.6 \\
\hline 15 & 5.7 & 61.4 & 2.9 & 49.8 & 3.7 & 21.4 \\
\hline 20 & 1.9 & 63.2 & 3.3 & 53.2 & 8.6 & 29.9 \\
\hline 25 & 2.8 & 66.1 & 2.9 & 56.1 & 10.7 & 40.6 \\
\hline 30 & 4.7 & 70.8 & 4.2 & 60.3 & 12.3 & 52.9 \\
\hline 35 & 8.5 & 79.3 & 5.9 & 66.1 & 14.4 & 67.4 \\
\hline 40 & 4.7 & 84.0 & 9.2 & 75.4 & 10.7 & 78.1 \\
\hline 45 & 7.5 & 91.5 & 7.1 & 82.5 & 5.3 & 83.4 \\
\hline 50 & 0.9 & 92.5 & 5.4 & 87.9 & 0.5 & 84.0 \\
\hline 55 & 1.9 & 94.4 & 3.8 & 91.7 & 0.5 & 84.5 \\
\hline 60 & 3.8 & 98.1 & 8.4 & & 0.0 & 84.5 \\
\hline 65 & 0.0 & 98.1 & & & 0.0 & 84.5 \\
\hline 70 & 0.9 & 99.1 & & & 0.5 & 85.0 \\
\hline Adultos & 0.9 & & & & 15.0 & \\
\hline Totales & 100.0 & 100.0 & 100.0 & 100.0 & 100.0 & 100.0 \\
\hline
\end{tabular}

CuADro 3. Distribución porcentual por grupos de edad quinquenales.

Fuente: Análisis osteológico y cálculos propios.

Los Datos de Copán proceden de la base de datos

de Márquez y Storey (1996).

Análisis de adultos

Una controversia que surge en los trabajos paleodemográficos es la escasa representación de los individuos adultos mayores de 50 años. ${ }^{9}$ Se argumenta que no

${ }^{9}$ Los primeros estándares utilizados para estimar la edad llegaban hasta la edad de 50 años, dejando fuera a los que probablemente habían alcanzado más de cincuenta años al morir; en la propuesta del "Análisis transicional" (Bolsen et al., 2002) se propone una técnica estandarizada para estimar edad en adultos mayores. 
se utilizan las técnicas adecuadas para estimar edades avanzadas, o bien debido al mal estado de conservación de los materiales. Otra interpretación es que los individuos morían antes de los 60 años. En el caso de Jaina, el porcentaje de adultos de 50 años y más es del $8 \%$, mientras que en Palenque es del $16 \%$ y en Copán del 13\%. Estos porcentajes tienen relación con dos aspectos: el primero, que ya ha sido discutido, es la conformación de la serie, y el segundo son los factores que determinaron la sobrevivencia de los individuos, que en este caso fueron las enfermedades infecciosas, pues, de acuerdo con la bibliografía consultada sobre el estado de salud de estos individuos, la mayoría tenía al morir algún tipo de padecimiento infeccioso activo. ${ }^{10}$

\section{La metodología paleodemográfica}

Las principales críticas al trabajo paleodemográfico consisten en la representatividad de la muestra, ya que uno de los supuestos básicos de esta técnica es que la distribución por edades a la muerte de los individuos de un cementerio o de un sitio arqueológico esté compuesta por los individuos que vivieron en ese lugar (Bocquet-Appel, 1985 y 1996; Bocquet-Appel y Masset, 1982). Por otra parte, también se cuestiona la estimación de la edad a partir de series de referencia, diferentes a la serie blanco (Hoppa y Vaupel, 2002). De ahí todo el procedimiento de evaluación de la calidad de las muestras que describimos en los párrafos anteriores.

Una vez definidas las tres series: Jaina, Palenque y Copán, se procedió a ajustar el número de menores de 15 años, únicamente para Palenque, por lo que se agregaron los individuos faltantes hasta que su número representara el $30 \%$ del total de la serie. ${ }^{11}$

En cuanto al factor asociado al crecimiento de la población, se considera que el trabajo paleodemográfico no se puede realizar bajo el supuesto de poblaciones con crecimiento cero, pues el comportamiento demográfico de las sociedades a través del tiempo ha demostrado que hay fluctuaciones. Los grupos algunas veces decaen; otras, crecen, de ahí que nos basamos en la información arqueológica para calcular la tasa de crecimiento para cada serie de acuerdo con sus circunstancias históricas específicas (Meindl, 2003; Meindl et al., 2008). Para Copán y Jaina fijamos una tasa de crecimiento de $0.015,{ }^{12}$ y en el caso de Palen-

\footnotetext{
10 Para mayor información consultar Storey, 1997 y 1999, y Márquez y Hernández, 2006a, 2006b y 2007.

${ }^{11}$ Meindl y Russell (1998) recomiendan la adición directa de recién nacidos y menores de cinco años para compensar la ausencia de aquellos infantes que no fueron recuperados de la excavación o enterrados en el sitio excavado, y que fueron víctimas de infanticidio. Por otro lado, Livi-Bacci (2002: 137-138) señala que entre las poblaciones antiguas el 30\% de los nacimientos anuales no llegaban a cumplir el primer aniversario de vida.

${ }^{12}$ La información arqueológica que disponemos de Jaina (Benavides, 2007) y de Copán (Webster et al., 2000) sugiere una población con crecimiento demográfico, tanto social como natural; una tasa de crecimiento menor no podría explicar el número de individuos infantiles representados en ambas series, lo que, según Konigsberg y Frakenberg (2002), es un indicador de alta fecundidad y, por lo
} 
que, de 0.005 , ya que esta población estaba en descenso en el periodo analizado (Liendo Stuardo, 2004).

La metodología estadística utilizada para la obtención de tasas e índices paleodemográficos fue propuesta por Kenneth W. Weiss (1973), y está basada en la distribución por edades a la muerte de los individuos conforme a los esqueletos recuperados de un cementerio o de las excavaciones arqueológicas. A partir de esta distribución se construye la tabla de mortalidad, que es una herramienta estadística que resume el impacto de la mortalidad en una población en un determinado momento en el tiempo. Una vez construida la tabla de mortalidad es posible obtener el resto de los indicadores paleodemográficos (ver anexo al final del artículo).

\section{Descripción de resultados: la mortalidad, la sobrevivencia y la fecundidad}

El crecimiento demográfico depende de los nacimientos y las defunciones ocurridas en una población en un tiempo determinado. Se mide obteniendo la diferencia algebraica de los valores de las tasas brutas de mortalidad y natalidad, e indica grosso modo los niveles alcanzados por estos dos fenómenos demográficos, sin considerar la estructura por edad ni el sexo de la población.

De las tres series seleccionadas, los resultados de los valores calculados sugieren que Jaina tendría los valores más altos, con 63.9 nacimientos y 43.9 defunciones por cada mil habitantes seguidos por tasas que oscilan entre 39 nacimientos y 24 defunciones por cada mil para Palenque, y de 38.3 y 23.3 para Copán (cuadro 4).

\begin{tabular}{|c|c|c|c|}
\hline Indicadores* & Palenque & Copán & Jaina \\
\hline TBN & 39.1 & 38.3 & 63.9 \\
\hline TBM & 24.1 & 23.3 & 43.9 \\
\hline $\mathrm{q}_{(0)}$ & 133.0 & 233.0 & 333.0 \\
\hline
\end{tabular}

CuADro 4. Tasas brutas de mortalidad y natalidad. Fuente: cálculos propios.

tanto, de crecimiento demográfico. Por otro lado, Hernández y Márquez (2007) realizaron el análisis paleodemográfico de Jaina, simulando varios escenarios demográficos. Aquel que se construyó con una tasa de 0.015 es congruente con el perfil cultural de dicha población.

* TBN: Tasa bruta de natalidad por cada mil individuos.

TBM: Tasa bruta de mortalidad por cada mil individuos.

$\mathrm{q}_{(0)}$ : Tasa de mortalidad infantil por mil nacimientos. 
El número de nacimientos calculados para Jaina es similar al de aquellas poblaciones con régimen de fecundidad natural, ${ }^{13}$ como las sociedades Hutteritas ${ }^{14}$ cuyas mujeres tenían un promedio de 12 hijos nacidos vivos. Entre las sociedades cazadoras-recolectoras actuales están los Ache de Paraguay, de quienes se reporta una tasa de nacimientos para mediados del siglo xx similar a la de Jaina (Hill y Hurtado, 1996: 253). Este tipo de información casi no existe para grupos prehispánicos; sin embargo, en el caso de Puerto Rico, Curet (2005: 203) reporta para Punta Candelero y los asentamientos Tibes tasas de natalidad superiores a los 69 nacimientos por mil habitantes. De los grupos mayas contemporáneos, disponemos de información etnográfica, proporcionada por Daltabuilt Godás (1992), para las mujeres de Yalcobá, Yucatán, que reporta 63 nacimientos por cada mil habitantes.

Los valores calculados para Palenque y Copán se consideran moderados y podrían estar influidos por algún tipo de control de nacimientos o una alta mortalidad de las mujeres en edad reproductiva. La probabilidad de sobrevivencia a los 15 años $\left(l_{(15)}\right)$ calculada para estas poblaciones muestra valores bajos que significan que sólo la mitad de la población sobrevivió a esa edad, lo que podría explicar los niveles moderados de nacimientos. Por otro lado, en el caso Copán, Whittington (1989) encuentra una alta frecuencia de lesiones relacionadas con anemia entre las mujeres jóvenes, lo que, según el autor, habría afectado su capacidad reproductiva.

La tasa de mortalidad infantil es uno de los indicadores específicos que tienen estrecha relación con las condiciones materiales de existencia de una población y con la atención hacia la salud de la madre y del recién nacido. El valor más alto se calculó para Jaina, con 333 muertes de niños menores de un año, atendiendo al planteamiento de Konigsberg y Frakenberg (2002), quienes señalan que una alta representación de menores de cuatro años en un sitio de enterramiento en realidad revela altos niveles de fecundidad. Una de las características demográficas de sociedades prehistóricas es la alta mortalidad infantil; entre los recién nacidos era de casi el 30\%, y entre los menores de cinco años alcanzaba cifras equivalentes al $40 \%$ de los efectivos sobrevivientes en ese rango de edad como consecuencia del destete y del proceso de ablactación (gráfica 1) (Livi-Bacci, 2002; Malgosa, 2010).

${ }^{13}$ Louis Henry (1953) llevó a cabo un estudio sobre fecundidad entre sociedades históricas y contemporáneas que no practicaban la anticoncepción. El autor definió como fecundidad no controlada al comportamiento reproductivo de mujeres o de parejas que no practicaban ningún tipo de anticoncepción, sin importar el número de hijos que tuvieran; en contraste, la fecundidad controlada se refiere al comportamiento de aquellas mujeres o parejas que, dependiendo del número de hijos, utilizaban algún medio de contracepción.

14 Son grupos endogámicos asentados en algunos estados del centro y norte de los Estados Unidos; proceden de grupos endogámicos europeos que viven en Alemania y en los Países Bajos. Su estilo de vida está dirigido al bienestar físico de sus mujeres, quienes están convencidas de que su papel es la reproducción, por lo que una mujer puede llegar a tener hasta 18 hijos vivos y gozar de excelente salud (para mayor información véase a Wood, 1994). 


\section{Indicadores de sobrevivencia}

La esperanza promedio de vida al nacimiento expresa la duración media de la vida de una generación de nacimientos y es una función de la intensidad de la mortalidad en las diversas edades (Livi-Bacci, 2002: 17-20). Tradicionalmente se consideró dentro de los indicadores de mortalidad, pero, en virtud de que tiene más relación con los límites naturales de la vida humana y los factores de desarrollo social, económico y tecnológico, es más lógico considerarlo un indicador de sobrevivencia, al igual que el promedio de edad de toda la población (A) y de la población adulta (AA), así como el porcentaje de sobrevivientes después de los 50 años (S) (cuadro 5).

\begin{tabular}{|c|c|c|c|}
\hline Indicadores* $^{*}$ & Palenque & Copán & Jaina \\
\hline $\mathrm{E}_{(0)}$ & 25.5 & 26.1 & 15.6 \\
\hline $\mathrm{A}$ & 20.5 & 26.0 & 19.5 \\
\hline $\mathrm{AA}$ & 31.6 & 37.1 & 31.6 \\
\hline $\mathrm{S}$ & 19.0 & 39.9 & 19.0 \\
\hline
\end{tabular}

CuAdro 5. Indicadores de sobrevivencia. Fuente: cálculos propios.

Copán tiene los valores más altos en los cuatro indicadores, ${ }^{15}$ seguido de Palenque y Jaina (cuadro 4), con valores semejantes en las edades promedio; respecto a la esperanza promedio de vida al nacimiento presenta una diferencia de 10 años con Jaina, como consecuencia de la alta mortalidad infantil reportada para esta serie (Hernández Espinoza y Márquez Morfín, 2007). Las diferencias encontradas entre estos dos grupos tienen una alta significación estadística (p $=0.001$ ) cuya explicación reside en el cambio de condiciones de vida y en su organización social, que se explicarán más adelante.

\section{Indicadores de fecundidad}

La unidad de medida de la fecundidad se expresa en hijos nacidos vivos. En paleodemografía esta medida se define como el promedio de hijos nacidos por mujer

${ }^{15}$ Ortega Muñoz (2007) reporta para el asentamiento de El Meco, Quintana Roo, una esperanza promedio de vida al nacimiento de 26.8 años. El análisis osteológico muestra una alta frecuencia de padecimientos infecciosos que afectaron la duración de la vida de los individuos que integraron la muestra.

* $\mathrm{E}_{(0)}$ : Esperanza promedio de vida al nacimiento.

A: Edad promedio de los individuos de la serie cuando estaban vivos.

AA: Edad promedio de los mayores de 15 años cuando estaban vivos.

S: Porcentaje de individuos que sobrevivieron los 50 años. 
que sobrevivió hasta los 50 años (final de su vida reproductiva), también es denominada Tasa global de fecundidad. Al considerar la mortalidad en la construcción de esta tasa, se transforma en el indicador F, o tamaño promedio de familia, que expresa el número de hijos e hijas sobrevivientes hasta los 15 años, o sea, el número de hijos que sobrevivieron para reproducirse.

Las tasas brutas y netas de reproducción señalan el número de niñas que haya tenido una mujer a lo largo su vida reproductiva. La diferencia entre una y otra es que la última es una tasa intervenida por la mortalidad y significa el número de hijas que cada niña recién nacida tendría para reemplazar a su generación. La importancia en el número de niñas es vital en demografía, significa el reemplazo generacional. Una mujer debería tener al menos una niña sobreviviente hasta los 15 años, que sería su reemplazo generacional.

Al analizar los resultados de estos indicadores en las series del periodo Clásico, Jaina tiene los valores más altos, mientras que Palenque y Copán presentan valores idénticos. Los resultados obtenidos en el caso de Jaina apoyan lo que hemos sugerido con anterioridad, que su población tuvo un régimen de fecundidad natural y que representa uno de los ejemplos más evidentes de sociedades bajo el régimen de alta presión demográfica. ${ }^{16} \mathrm{El}$ indicador T Barra es un número que oscila entre 26 o 27 años para poblaciones antiguas y se asocia con la duración del periodo reproductivo (Livi-Bacci, 2002; Meindl y Russell, 1998; Weiss, 1973: 7). Los resultados de las tres series presentan valores dentro de este rango (cuadro 6). La tasa neta de reproducción $\left(\mathrm{R}_{(0)}\right)$ mantiene valores entre 1.5 y 1.7 niñas por mujer, lo que implica que el reemplazo generacional estaba asegurado. Estos valores son también indicativos de una población con crecimiento moderado.

\section{Reproducción y sobrevivencia}

La capacidad de crecimiento de una población puede expresarse en función de dos medidas: el número de nacimientos o hijos por mujer y la esperanza de vida al nacimiento. Se trata de las medidas de reproducción y sobrevivencia, respectivamente. La primera expresa el número promedio de hijos dados a luz por una generación de mujeres a lo largo de su vida reproductiva, bajo el supuesto teórico de ausencia de la mortalidad; la segunda, como ya se mencionó, es una función de la intensidad de la mortalidad en las diversas edades. Ambos indicadores, dependiendo de la influencia de los factores culturales, biológicos, socioeconómicos y ambientales, podría alcanzar valores distintos; por ejemplo, entre las poblaciones antiguas el número de hijos por mujer podía variar de menos de

\footnotetext{
${ }^{16}$ Wood (1990) estudió la fecundidad en 70 sociedades tradicionales, encontrando que en el 90\% de los casos el número de hijos nacidos vivos que cada mujer tuvo a lo largo de su vida reproductiva oscilaba entre cuatro y ocho, con un número promedio de seis.
} 


\begin{tabular}{|c|c|c|c|}
\hline Indicadores* $^{*}$ & Palenque & Copán & Jaina \\
\hline TGF & 5.0 & 5.0 & 8.8 \\
\hline TBR & 2.5 & 2.5 & 4.4 \\
\hline $\mathrm{R}_{(0)}$ & 1.5 & 1.5 & 1.5 \\
\hline T barra & 26.7 & 27.6 & 26.7 \\
\hline $\mathrm{F}$ & 2.9 & 3.7 & 5.0 \\
\hline$l_{(15)}$ & 0.7000 & 0.5410 & 0.4340 \\
\hline
\end{tabular}

CuAdro 6. Indicadores de fecundidad. Fuente: cálculos propios.

cinco a más de ocho, y la esperanza de vida podría oscilar entre 20 y 40 años. Los factores que influyen en una sociedad son específicos de las circunstancias históricas, de ahí que no sea recomendable generalizar los hallazgos basados en una serie osteológica a otras poblaciones.

Jaina tiene los valores más altos en estos indicadores (cuadro 6), similares a los de las mujeres mayas actuales que habitan en la selva lacandona, los cuales reportan un promedio de 8.7 hijos nacidos vivos, hasta una vez alcanzada la edad de la menopausia (Daltabuilt Godás et al., 1994). Para las mujeres mayas de Yalcobá, Yucatán, en edades entre los 45 y 49 años, se reportó un promedio de 8.1 hijos nacidos vivos. Entre los grupos cazadores recolectores actuales la fecundidad más alta se reporta para los ache y los yanomame, que habitan en las selvas de América del Sur; las mujeres en edades de menopausia tuvieron un total de 7.9 hijos nacidos vivos, haciendo la aclaración de que el mayor número de hijos sobrevivientes procede de las mujeres que rebasaron los 40 años. La TBR señala el número de hijas que, a su vez, tienen las mujeres para garantizar su reemplazo generacional; en términos demográficos, este indicador es igual a dos, por lo que en el caso de las tres series tuvieron asegurado su reemplazo generacional.

Un indicador interesante para el análisis es la edad media a la fecundidad (T barra), que Weiss (1973) interpreta como la duración del periodo reproductivo. Es difícil establecer si los 26-27 años calculados para estas series corresponden a la duración de los años fértiles femeninos, dado que, por un lado, este periodo

* TGF: Tasa global de fecundidad.

TBR: Tasa bruta de reproducción.

$\mathrm{R}_{(0)}$ : Tasa neta de reproducción.

T: Duración aproximada del periodo reproductivo.

F: Número de hijos que sobrevivieron a la edad de 15 años.

$l_{(15)}$ : Probabilidad de sobreviviencia a la edad de 15 años. 
está interferido por la salud de las mujeres y la escasa sobrevivencia femenina hasta los 50 años, y, por el otro, la forma de cálculo del indicador tiene una mayor relación con una edad promedio, por lo cual preferimos interpretarla como la edad promedio en que las mujeres de las series tuvieron a sus hijos, lo que coincide con lo que señala Ortega Muñoz (2007) y nosotras mismas para los casos de Palenque y de Jaina, en donde la mayor fecundidad se manifiesta entre los 20 y los 29 años de edad (Hernández Espinoza y Márquez Morfín, 2005; Márquez Morfín y Hernández Espinoza, 2004).

\section{Discusión}

En síntesis, con relación a los resultados obtenidos del estudio demográfico podemos describir los siguientes perfiles. Las series de Copán y Palenque tienen un comportamiento demográfico similar, con tasas de mortalidad moderadas y una alta fecundidad que resultó en una alta densidad poblacional. Indicadores más refinados de la mortalidad, como la infantil y la sobrevivencia a edades mayores de 50 años, marcan diferencias entre las dos urbes. Copán tiene una tasa de mortalidad infantil más alta y un mayor porcentaje de adultos mayores de 50 años, aunque la esperanza de vida al nacimiento es casi similar a la de Palenque. La tasa de mortalidad infantil en los centros urbanos es el resultado del hacinamiento y de la simbiosis de los procesos infecciosos, provocados por el consumo de agua y alimentos contaminados, con el clima caliente y húmedo de las zonas tropicales (Márquez et al., 2001).

En Palenque, los valores de estos indicadores son más bajos, ya que la muestra, al igual que en el caso de Copán, procede de un sector privilegiado de la población que habitó esta ciudad, y, aunque los procesos infecciosos provocados por problemas de sanidad pública afectaban por igual a la elite y a los comunes, los primeros tenían ciertas ventajas sobre los segundos, como el acceso a una buena alimentación, que, de algún modo, reforzaba el sistema inmune, y el no verse sometidos a grandes cargas de trabajo. En cuanto a la sobrevivencia de los adultos mayores de 50 años, creemos que las diferencias se deben a la composición de la muestra, pues mientras que en Copán las exploraciones han cubierto gran parte del sitio, en Palenque no fue así, y el adulto más longevo de la serie no rebasa los 60 años.

El caso de Jaina es interesante, pues su modo de subsistencia estaba basado en el consumo de recursos naturales que provenían del mar. El crecimiento y la densidad de población hacia el periodo de mayor auge pudo haber provocado serios problemas de salud entre sus habitantes, en particular enfermedades infecciosas. La tasa de mortalidad infantil en Jaina es la mayor de las series aquí analizadas, al igual que la mortalidad mostrada para el caso de Tlajinga 33, el barrio teotihuacano. Los valores tan altos para ambos casos supondrían un rápido crecimiento de la población; sin embargo, habría que recordar que dicho crecimiento no era sostenido, como sucede en la época actual. Por lo general, después de un periodo de aumento demográfico, seguía uno de contracción, provocado por catástrofes naturales, en- 
fermedades o guerras, como se ha documentado para el caso de las poblaciones europeas del antiguo régimen ${ }^{17}$ como consecuencia de las condiciones precarias de existencia y de las infecciones. Además, debemos considerar los problemas ocasionados por el destete y el proceso de ablactación, que bien pudo haber sido problemático en estas regiones mesoamericanas. Una consecuencia de la alta mortalidad a edades jóvenes es la baja esperanza promedio de vida al nacimiento y, por lo tanto, la escasa sobrevivencia a edades mayores. También la alta fecundidad es característica de los regímenes de alta presión ${ }^{18}$ como el de Jaina, cuyo lento crecimiento fue producto de una gran disipación de energía demográfica. Siguiendo la idea de Livi-Bacci (2002: 137-138), las mujeres debían dar a luz una media docena de hijos para poder ser reemplazadas por la generación posterior. Cada generación de nacidos perdía de la mitad a una tercera parte de sus componentes antes de que estos alcanzasen la edad reproductiva y pudieran convertirse en reproductores, lo que supone que, en Jaina, de casi nueve hijos e hijas nacidos por mujer, sólo cinco sobrevivieron para reproducirse. En general, de acuerdo a la información arqueológica, se sabe que la sociedad maya era pronatalista, con tendencia al matrimonio universal a edad temprana. Entre la elite era común tener varias mujeres, lo que aumentaba la probabilidad de engendrar muchos hijos sobrevivientes.

La interpretación integral de la información paleodemográfica permitió detectar la dinámica demográfica en estas tres series de población maya. Jaina tuvo un modo de subsistencia basado en la pesca, complementado con el consumo de alimentos derivados del maíz. Sus niveles de mortalidad y de fecundidad la sitúan entre las sociedades con un régimen demográfico de alta presión. La mortalidad infantil y durante la niñez es de las más altas calculadas para una población maya que apunta hacia problemas derivados de procesos infecciosos y respiratorios durante el primer año de vida, y de adaptación al medio ambiente en la época del destete y de ablactación, que, de acuerdo con la información osteológica, debió ocurrir alrededor de los tres años de edad. Los indicadores de fecundidad señalan de siete a nueve hijos en promedio por mujer; sin embargo, este dato habría que tomarlo con reserva, pues, de acuerdo con las probabilidades de muerte calculadas para esta serie, una buena proporción de los individuos tendrían una escasa probabilidad de sobrevivencia al inicio del periodo productivo y reproductivo. ${ }^{19}$

${ }^{17}$ El antiguo régimen demográfico europeo (1500 a 1820) se caracterizó por tener niveles de mortalidad y fecundidad altos y sin control, con un crecimiento oscilante y lento de la población. El determinante de más peso es lo que Flinn (1989: 74-97) llama la inestabilidad de la mortalidad, que rompía el frágil equilibrio demográfico.

18 "Las sociedades del antiguo régimen eran, por consiguiente, ineficientes. Desde el punto de vista demográfico, para obtener un nivel bajo de crecimiento necesitaban abundante combustible (los nacimientos) y dispersaban una enorme cantidad de energía producida (los muertos). Además de ineficiente, el antiguo régimen demográfico era ‘desordenado'. Las probabilidades de que un hijo muriese antes que su padre eran muy altas, el orden natural se invertía” (Livi-Bacci, 2002: 137).

${ }^{19}$ De acuerdo con los datos proporcionados al inicio de este trabajo, el $50 \%$ de los individuos representados en la serie tenían al morir menos de diez años, lo que supone que menos de la mitad de 
Palenque y Copán, dos de los centros urbanos más importantes del Clásico maya, con una alta densidad de población, tienen problemas de salud derivados de la falta de higiene pública y del hacinamiento. Los niveles de mortalidad y fecundidad habrían sido similares y con un crecimiento demográfico moderado, de acuerdo con los datos calculados a partir de la distribución por edades de ambas series, por lo que deben tomarse como un escenario hipotético, sin generalizarse a toda la población. La serie de Palenque representa sólo a un sector de sus habitantes: la elite secundaria, y en el caso de Copán también, pues la colección ósea fue recuperada de la Casa de los Bacabes, uno de los barrios de mayor prestigio de la ciudad. Como sociedades del antiguo régimen demográfico, los factores culturales tales como la lactancia prolongada, el proceso de ablactación, la edad al matrimonio y la poliginia, son factores que median en el crecimiento demográfico y, por ende, en los niveles de fecundidad (Hernández, 2006; Storey et al., 2002: 281-306).

El análisis paleodemográfico resulta todo un reto para los estudiosos de este tipo de temas, por las múltiples limitantes antes mencionadas, pues es probable que los individuos integrantes de las series esqueléticas provengan de momentos de crecimiento o de contracción demográfica, por lo que interpretar cifras y datos es más complicado que calcularlos. Sin embargo, el análisis de los esqueletos es la única fuente de información para conocer la dinámica demográfica de los grupos antiguos. Los resultados obtenidos son un ejemplo del esfuerzo sostenido durante más de dos décadas de investigación paleodemográfica, con el objetivo de entender la relación entre la sociedad, la salud, los aspectos sociales, económicos y políticos, y su impacto en la mortalidad, la sobrevivencia, la esperanza de vida y la fecundidad. El enfoque biosociocultural es esencial en este tipo de estudios, que pretenden conocer dichos fenómenos de manera integral.

El objetivo central es describir, analizar y proporcionar interpretaciones alternativas de una forma sencilla sobre los patrones demográficos y la manera de calcularlos; no obstante, para los interesados en esta metodología, es necesario recurrir a textos específicos donde se abunde en las cuestiones teóricas, metodológicas y técnicas necesarias para la realización de estas investigaciones.

los individuos de la serie sobrevivieron a los 15 años (cuadro 3), edad que convencionalmente marca el inicio de la vida productiva y activa en las sociedades antiguas; sin embargo, habría que señalar que estos cálculos son sólo para la serie analizada y no para generalizar a toda la población maya. 


\section{Anexo}

Indicadores paleodemográficos utilizados en este capítulo de acuerdo con Weiss 1973.

$A=$ promedio de edad de toda la población $=\frac{\sum_{0}^{80} \bar{x} \hat{\mathrm{C}}_{(x)}}{\sum_{0}^{80} \hat{C}_{(x)}}$

$A A=$ edad promedio de la población adulta (mayor de 15 años) $=\frac{\sum_{15}^{80} \bar{x} \hat{C}_{(x)}}{\hat{C}(15, \bar{\omega})}$

$S=$ porcentaje de sobrevivientes a edades mayores de 50 años $=100\left[\frac{l_{(50)}}{l_{(15)}}\right]$

TBN = tasa bruta de natalidad $=b=1 / \sum_{0}^{80} e^{\overline{r x}}$ Lest,

$T B M=$ tasa bruta de mortalidad $=b-r$

$q_{(\mathrm{o})}=$ tasa de mortalidad infantil $=\frac{\mathrm{d}_{(0)}}{l_{(0)}}$

$C_{(x)}=$ Censo hipotético de población $=\frac{L_{(x)}}{T_{(x)}}$

$\bar{b}=(\mathrm{b}$ barra $)$ tasa intrínseca de fecundidad $=\frac{1}{\sum{\text { Lest' } \mathrm{K}_{\mathrm{x}}}}$

$f x=$ tasas específicas de fecundidad $=K_{(\mathrm{x})} \bar{b}$

$T B R=$ tasa bruta o reproducción o número de hijas promedio por mujer $=\sum_{15}^{50} 5_{f_{x}}$ 
$T G F=$ tasa global de fecundidad o promedio de hijos e hijas promedio por mujer $=T B R * 2$

$R_{(0)}=$ tasa neta de reproducción o número de hijas que sobrevivieron hasta el inicio de la edad reproductiva para reemplazar a su generación $=\sum$ Lest' $f_{(x)}$

$\bar{T}=$ (t barra) duración del periodo reproductivo o tambien llamado de duración de la generación $=\frac{\mathrm{R}_{0}}{r}$

$F=$ hijos e hijas sobrevivientes al inicio de la edad reproductiva $=\frac{2 R_{0}}{\hat{l}_{15}}$

\section{BIBLIOGRAFÍA}

Andrews, Anthony P., Tomás Gallareta N., Fernando Robles C., Rafael Cobos P. y Pura Cervera $\mathrm{R}$.

1988 "Isla Cerritos: An Itzá Trading Porth on the North Coast of Yucatán, México”, National Geographic Research, 4: 196-207. Cambridge: National Geographic Society.

Andrews, Anthony P. y Fernando Robles C.

1985 "Chichen Itza and Coba: An Itza Maya Standoff in Early Postclassic Yucatan", The Lowland Maya Postclassic, Arlen F. Chase y Prudence M. Rice (eds.). Austin: Texas University Press.

Bass, William

1995 Human Osteology: A Laboratory and Field Manual of the Human Skeleton. Columbia: University of Missouri.

Benavides, Antonio

2007 "Jaina en el contexto de las poblaciones del Clásico en el occidente peninsular”, La población prehispánica de Jaina. Estudio osteobiográfico de 106 esqueletos, Patricia Olga Hernández Espinoza y Lourdes Márquez Morfín (eds.), pp: 13-32. México: Instituto Nacional de Antropología e Historia, Escuela Nacional de Antropología e Historia / Secretaría de Educación Pública, Programa de Mejoramiento del Profesorado.

Bocquet-Appel, Jean Pierre

1985 "Paleodemography: Resurrection or Ghost?", Journal of Human Evolution, 14: 107-111. San Diego: Elsevier.

1996 "Paleodemography: Expectancy and False Hope", American Journal of Physical Anthropology, 99: 571-583. Hoboken: Wiley-Blackwell.

Bocquet-Appel, Jean Pierre y Claude Masset

1982 "Farewell to Paleodemography", Journal of Human Evolution, 11: 321-333. San Diego: Elsevier. 
Buikstra, Jane E. y Douglas H. Ubelaker

1994 Standards for Data Collection From Human Skeletal Remains. Fayetteville: Arkansas Archaeological Survey.

Chacín, Nadir

2003 "Aculturación religiosa y control culural a través del ritual funerario maya: la capilla abierta de Xcaret durante los siglos xvı y xvI, Quintana Roo, México", tesis de Maestría en Antropología Física. México: Escuela Nacional de Antropología e Historia.

Cobos Palma, Rafael

2004 "Chichen Itza. Settlement and Hegemony during the Terminal Classic Period”, The Terminal Classic in the Maya Lowlands. Collapse, Transition and Transformations, Arthur A. Demarest, Prudence M. Rice y Don S. Rice (eds.), pp: 517-544. Boulder: The University Press of Colorado.

Cohen, Mark Nathan y Gillian M. M. Crane-Kramer

2007 Ancient Health. Skeletal Indicators of Agricultural and Economic Intensification. Gainesville: University Press of Florida.

Con, María José

1991 Informe del Proyecto Xcaret. Cuarta y quinta temporada, 1990-1991. México: Instituto Nacional de Antropología e Historia.

Curet, L. Antonio

2005 Caribbean Paleodemography. Population, Cultural History and Sociopolitical Processes in Ancient Puerto Rico. Tuscaloosa: The University of Alabama Press.

Daltabuilt Godás, Magalí, Luz María Vargas, Enrique Santillán y Héctor Cisneros

1994 "Mujeres de la Selva Lacandona", Mujer rural y medio ambiente en la Selva Lacandona, Magalí Daltabuit Godás (ed.), pp: 59-77, Cuernavaca, Morelos: Universidad Nacional Autónoma de México, Centro Regional de Investigaciones Multidisciplinarias.

Daltabuit Godás, Magalí

1992 Mujeres mayas. Trabajo, nutrición y fecundidad. México: Universidad Nacional Autónoma de México, Instituto de Investigaciones Antropológicas.

Fascio Guillén, Helena Atenea

2003 "Determinación de las condiciones de salud y nutrición de los habitantes del Grupo IV de Palenque, Chiapas", tesis de Licenciatura en Antropología Física. México: Escuela Nacional de Antropología e Historia.

Flinn, Michael W.

1989 El sistema demográfico europeo, 1500-1820. Barcelona: Editorial Crítica (Historia y Teoría). 
Giannisis, Dimitrios

2006 "Patrones de actividad y organización social en la población costera maya de Chac Mool: estudio basado en los marcadores de estrés musculoesquelético”, La población maya costera de Chac Mool, Quintana Roo. Perfil biocultural y dinámica demográfica, Lourdes Márquez Morfín, Patricia Olga Hernández Espinoza y Ernesto González Licón (eds.), pp. 191-216. México: Instituto Nacional de Antropología e Historia, Escuela Nacional de Antropología e Historia (Publicaciones de los Cuerpos Académicos).

González Licón, Ernesto y Rafael Cobos Palma

2006 "El entorno sociopolítico de Chac Mool, Quintana Roo, durante el Clásico Terminal y el Posclásico", La población costera maya de Chac Mool. Perfil biocultural y dinámica demográfica, Lourdes Márquez Morfín, Patricia Olga Hernández Espinoza y Ernesto González Licón (eds.), pp: 27-45. México: Instituto Nacional de Antropología e Historia, Escuela Nacional de Antropología e Historia (Publicaciones de los Cuerpos Académicos).

González Licón, Ernesto y Enrique Terrones González

2003 "Género y desigualdad social entre los mayas de Chac-Mool, Quintana Roo, durante el Posclásico, a través de sus prácticas funerarias", ponencia presentada en el XII Coloquio Internacional de Antropología Física "Juan Comas". Tlaxcala: Asociación Mexicana de Antropología Biológica.

2004 "Gender, Hierarchy and Funerary Practices among Posclassic Maya of Chac Mool, Quintana Roo”, paper presented at 69th Annual Meeting. Montreal: Society for American Archaeology.

Goodman, Allan H. y Debra L. Martin

2002 "Reconstructing Health Profiles from Skeletal Remains", The Backbone of History. Health and Nutrition of the Western Hemispher, Richard H. Steckel y Jerome C. Rose (eds.), pp: 11-60. New York: Cambridge University Press.

Haviland, William A.

2003 "Settlement, Society and Demography at Tikal", Tikal: Dynasties, Foreigners \& Affairs of State, Jeremy. A. Sabloff (ed.), pp: 111-142. Santa Fe: School for American Research Press.

Henry, Louis

1953 "Fondements théoriques des mesures de la fécondité naturelle", Revue de l'Institut International de la Statistique, 3: 135-151. Paris: International Statistical Institute.

Hernández Espinoza, Patricia Olga

2006 La regulación del crecimiento de la población en el México prehispánico. México: Instituto Nacional de Antropología e Historia (Colección Divulgación).

Hernández Espinoza, Patricia Olga y Lourdes Márquez Morfín

2005 "Las mujeres de Jaina y su fecundidad", ponencia presentada en el XIII Coloquio Internacional de Antropología Física "Juan Comas". Campeche: Asociación Mexicana de Antropología Biológica. 
2006a "La población maya prehispánica. Una interpretación sobre su dinámica demográfica", Salud y sociedad en el México prehispánico y colonial, Lourdes Márquez Morfín y Patricia Olga Hernández Espinoza (eds.), pp: 103-126. México: Instituto Nacional de Antropología e Historia, Escuela Nacional de Antropología e Historia / Secretaría de Educación Pública, Programa para el Mejoramiento del Profesorado (Publicaciones de los Cuerpos Académicos).

2006b "Fecundidad y sobrevivencia: perfil paleodemográfico de la población maya prehispánica de Chac Mool, Quintana Roo", La población costera maya de Chac Mool: Análisis biocultural y dinámica demográfica en el Clásico Terminal y el Posclásico, Lourdes Márquez Morfín, Patricia Olga Hernández Espinoza y Ernesto González Licón (eds.), pp: 81-112. México: Instituto Nacional de Antropología e Historia, Escuela Nacional de Antropología e Historia / Secretaría de Educación Pública, Programa para el Mejoramiento del Profesorado (Publicaciones de los Cuerpos Académicos).

2007 "El escenario demográfico de Jaina prehispánica durante el Clásico", La población prehispánica de Jaina. Estudio osteobiográfico de 106 esqueletos, Patricia Olga Hernández Espinoza y Lourdes Márquez Morfín (eds.), pp: 33-62. México: Instituto Nacional de Antropología e Historia, Escuela Nacional de Antropología e Historia / Secretaría de Educación Pública, Programa para el Mejoramiento del Profesorado (Publicaciones de los Cuerpos Académicos).

Hernández Espinoza, Patricia Olga y María Eugenia Peña Reyes

2010 La identificación del sexo y la estimación de la edad en esqueletos de menores de 15 años (subadultos). México: Instituto Nacional de Antropología e Historia, Escuela Nacional de Antropología e Historia.

Hill, Kim y A. Magdalena Hurtado

1996 Ache Life History. The Ecology and Demography of a Foraging People. New York: Aldine de Gruyter / Foundations of Human Behavior.

Hoppa, Robert D. y James W. Vaupel

2002 Paleodemography. Age Distribution from Skeletal Samples. Cambridge: Cambridge University Press.

Konigsberg, Lyle W. y Susan R. Frankenberg

2002 "Deconstructing Death in Paleodemography", American Journal of Physical Anthropology, 117 (3): 297-309. Hoboken: Wiley-Blackwell.

Liendo Stuardo, Rodrigo

2000 "Reyes y campesinos. La población rural de Palenque”, Arqueología Mexicana 45: 34-37. México: Raíces.

2004 El paisaje urbano de Palenque: una perspectiva regional. México: Instituto Nacional de Antropología e Historia / Universidad Nacional Autónoma de México.

Livi-Bacci, Massimo

2002 Historia mínima de la población mundial. Barcelona: Editorial Ariel (Ariel Historia) 
López Alonso, Sergio y Carlos Serrano Sánchez

1984 "Prácticas funerarias en la isla de Jaina, Campeche", Investigaciones recientes en el Área Maya. Memoria de la XVII Mesa Redonda de la Sociedad Mexicana de Antropología, pp. 441-452. San Cristóbal de las Casas, Chiapas: Sociedad Mexicana de Antropología.

1997 "Los entierros humanos de Jaina, Campeche", El cuerpo humano y su tratamiento mortuorio, Elsa Malvido, Gregory Pereiya y Vera Tiesler (eds.), pp: 145-160. México: Instituto Nacional de Antropología e Historia.

2007 "Estatus social y contexto funerario durante el Clásico, en Jaina, Campeche”, La población prehispánica de Jaina. Estudio osteobiográfico de 106 esqueletos, Patricia Olga Hernández Espinoza y Lourdes Márquez Morfín (eds.), pp. 77-110. México: Instituto de Nacional de Antropología e Historia, Escuela Nacional de Antropología e Historia.

López Bravo, Roberto

1995 "El grupo B de palenque, Chiapas. Una unidad habitacional maya del Clásico Tardío”, tesis de Licenciatura en Arqueología. México: Escuela Nacional de Antropología e Historia.

2000 "La veneración de los ancestros en Palenque". Arqueología Mexicana, 45: 3843. México: Raíces.

Lovejoy, Owen C.

1985 "Dental Wear in the Libben Population: its Functional Pattern and Rol in the Determination of Adult Skeletal Age at Death". American Journal of Physical Anthropology, 68 (1): 47-56. Hoboken: Wiley-Blackwell.

Lovejoy, O. C., Richard S. Meindl, Thomas Pryzbeck y Robert P. Mensforth

1985 "Chronological Metamorphosis of the Auricular Surface of the Ilium: A New Method for the Determination of Adult Skeletal Age at Death", American Journal of Physical Anthropology, 68 (1): 15-29. Hoboken: Wiley-Blackwell.

Malgosa, Asunción

2010 "Vida, enfermedad y muerte en la antiguiedad: lo que nos cuentan los esqueletos de los niños", Los niños, actores sociales ignorados. Levantando el velo, una mirada al pasado, Lourdes Márquez Morfín (ed.), pp: 21-50. México: Instituto Nacional de Antropología e Historia, Escuela Nacional de Antropología e Historia / Proa.

Márquez Morfín, Lourdes y Ernesto González Licón

2010 "La socialización de los niños en el pasado. Algunas reflexiones y propuestas en torno al tema”, Los niños, actores sociales olvidados. Levantando el velo, una mirada al pasado, Lourdes Márquez Morfín (ed.), pp: 50-73. México: Instituto Nacional de Antropología e Historia, Escuela Nacional de Antropología e Historia / Proa.

Márquez Morfín, Lourdes, María Teresa Jaén Esquivel y José Concepción Jiménez López 2002 "Impacto biológico de la colonización en Yucatán. La población de Xcaret, Quintana Roo, México”, Antropología Física Latinoamericana, 3 25-42. México, 
Universidad Nacional Autónoma de México, Instituto de Investigaciones Antropológicas.

Márquez Morfín, Lourdes y Patricia Olga Hernández Espinoza

2004 "Aspectos sociodemográficos de la población maya de Palenque durante el Clásico Terminal a través del análisis de las costumbres funerarias y el dato bioarqueológico", Culto funerario en la sociedad maya, Memoria de la Cuarta Mesa Redonda de Palenque, Rafael Cobos Palma (ed.), pp. 415-442. México: Instituto Nacional de Antropología e Historia.

2006a "Los mayas prehispánicos. Balance de salud y nutrición en grupos del Clásico y el Posclásico", Salud y sociedad en el México prehispánico y colonial, Lourdes Márquez Morfín y Patricia Olga Hernández Espinoza (eds.), pp: 73-102. México: Instituto Nacional de Antropología e Historia, Escuela Nacional de Antropología e Historia / Secretaría de Educación Pública, Programa para el Mejoramiento del Profesorado (Publicaciones de los Cuerpos Académi$\cos )$.

2006b "¿Privilegios en la salud? Testimonio osteológico de un sector de la elite de Palenque", Sociedad y salud en el México prehispánico y colonial, Lourdes Márquez Morfín y Patricia Olga Hernández Espinoza (eds.), pp: 265-290. México: Instituto Nacional de Antropología e Historia, Escuela Nacional de Antropología e Historia / Secretaría de Educación Pública, Programa para el Mejoramiento del Profesorado (Publicaciones de los Cuerpos Académi$\cos )$.

2007 "Alimentación y Salud en algunos pobladores de Jaina, Campeche, durante el Clásico", La población prehispánica de Jaina. Estudio osteobiográfico de 106 esqueletos, Patricia Olga Hernández Espinoza y Lourdes Márquez Morfín (eds.), pp: 97-132. México: Instituto Nacional de Antropología e Historia, Escuela Nacional de Antropología e Historia / Secretaría de Educación Pública, Programa para el Mejoramiento del Profesorado (Publicaciones de los Cuerpos Académicos).

2008 "Población, salud y nutrición entre los mayas prehispánicos", El territorio maya. Memoria de la Quinta Mesa Redonda de Palenque, Rodrigo Liendo Stuardo (ed.), pp: 69-98. México: Instituto Nacional de Antropología e Historia.

Márquez Morfín, Lourdes, Patricia O. Hernández Espinoza y Ernesto González Licón 2001 "La salud en las grandes urbes prehispánicas”, Estudios de Antropología Biológica, X (1): 291-313. México: Universidad Nacional Autónoma de México, Instituto de Investigaciones Antropológicas.

Márquez Morfín, Lourdes, Robert McCaa, Rebecca Storey y Andrés del Ángel

2002 "Health and Nutrition in Pre-Hispanic Mesoamerica", The Backbone of History. Health and Nutrition in the Western Hemisphere, Richard H. Steckel y Jerome Rose (eds.), pp: 307-338. New York: The Cambridge University Press.

Meindl, Richard S.

2003 "Current Methodlogical Issues in the Study of Prehistoric Demography", Estudios de Antropología Biológica, XI: 679-692. México: Universidad Nacional Autónoma de México, Instituto de Investigaciones Antropológicas. 
Meindl, Richard S. y Katheryn F. Russell

1998 "Recent Advances in Method and Theory in Paleodemography", Annual Review of Anthropology, 27: 375-399. Palo Alto, CA: Annual Reviews.

Meindl, Richard S., Robert P. Mensforth y Owen C. Lovejoy

2008 "Comentarios sobre los principales errores del trabajo paleodemográfico: el cálculo de la mortalidad promedio, la estructura por edad y la tasa de crecimiento anual. Un ejemplo del Ohio prehistórico, en Estados Unidos", Tendencias actuales de la Bioarqueología en México, Patricia Olga Hernández Espinoza, Lourdes Márquez Morfín y Ernesto González Licón (eds.), pp: 15-36. México: Instituto Nacional de Antropología e Historia, Escuela Nacional de Antropología e Historia / Secretaría de Educación Pública, Programa para el Mejoramiento del Profesorado (Publicaciones de los Cuerpos Académicos).

Nuñez Enríquez, Luis Fernando

2003 "Análisis del contexto funerario del sitio de Chac Mool, Quintana Roo", tesis de Licenciatura en Antropología. Puebla: Universidad de Las AméricasPuebla.

Ortega Muñoz, Allan

2007 Los mayas prehispánicos de el Meco. La vida, la muerte y la salud en la costa oriental de la Península de Yucatán. México: Instituto Nacional de Antropología e Historia (Colección Científica. Serie Antropología).

Peña Reyes, María Eugenia, Patricia Olga Hernández Espinoza y Lourdes Márquez Morfín 2007 "Estatus de crecimiento y condiciones de salud en los niños Jaina", La población prehispánica de Jaina. Estudio osteobiográfico de 106 esqueletos, Patricia Olga Hernández Espinoza y Lourdes Márquez Morfín (eds.), pp: 139-164. México: Instituto Nacional de Antropología e Historia, Escuela Nacional de Antropología e Historia / Secretaría de Educación Pública, Programa para el Mejoramiento del Profesorado (Publicaciones de los Cuerpos Académicos).

Sauvin-Dugerdil, Claudine

2006 "The Human Clock: Overview”, Human Clocks. The Biocultural Meaning of Age, Claudine Sauvin-Dugerdil, Henri Leridon y Nicholas Mascie-Taylor (eds.), pp: 1. London: Peter Lang. Population, Family and Society.

Sharer, Robert J.

1999 La civilización maya. México: Fondo de Cultura Económica.

Storey, Rebeca

1992a Life and Death in Ancient City in Teotihuacan. A Modern Paleodemographic Synthesis. Alabama: The University of Alabama Press.

1992b “The Children of Copán”, Ancient Mesoamerica, 3: 161-168. Cambridge: Cambridge University Press.

1997 "Individual Frailty, Children of Privilege and Stress in Late Classic Copan", Bones of the Maya, S. L. Whittington y D. M. Reed (eds.), pp: 116-126. Washington: Smithsonian Institution Press. 

constructing Ancient Maya Diet, C. White (ed.), pp: 169-182. Salt Lake City: University of Utah Press.

Storey, Rebeca, Lourdes Márquez y Vernon Schmidt

2002 "Social Disruption and the Maya Civilization of Mesoamerica: A Study of Health and Economy of the Last Thousand Years", The Backbone of History. Health and Nutrition in the Western Hemisphere, R. H. Steckel y J. C. Rose (eds.), pp: 281-306. New York: Cambridge University Press.

Stuart, George S. y G. E. Stuart

1993 Los reinos perdidos de los mayas. Barcelona: National Geographic.

Ubelaker, Douglas $\mathrm{H}$.

1989 "The Estimation of Age at Death from Immature Human Bone", Age Markers in the Human Skeleton, M. Y. Iscan (ed.), New York: Academic Press.

Webster, David, Ann Corinne Freter y Nancy Golin

2000 Copan. The Rise and Fall of an Ancient Maya Kingdom. Belmotn: WadswothGroup / Thomson Learning, First. Case Studies in Archaeology.

Weiss, Kenneth W.

1973 Demographic Models for Anthropology. Memoir 27. Washington: Society for American Archaeology.

Whittington, Stephen L.

1989 "Characteristics of Demography and Disease in Low Status Maya from Classic Period Copán, Honduras", Ph D. Dissertation. Pennsylvania: The Pennsylvania State University, University Park

1991 "Detection of Significant Demographic Differences Between Subpopulations of Prehispanic Maya from Copan, Honduras", American Journal of Physical Anthropology, 85 (2): 167-184. Hoboken: Wiley-Blackwell.

1992 "Enamel Hypoplasia in the Low Status Maya Population of Prehistoric Copan, Honduras", Journal of Paleopathology, 2 (1): 185-205. San Diego: Elsevier.

1999 "Caries and Antemortem Tooth Loss at Copán", Reconstructing Ancient Maya Diet, C. White (ed.), pp: 151-167. Salt Lake City: University of Utah Press.

Wood, James W.

1990 "Fertility in Anthropological Populations", Annual Review of Anthropology, 19: 211-242. Palo Alto, CA: Annual Reviews.

1994 “Natural Fertility. Introduction”, Dynamics of Human Reproduction, pp: 3-22. New York: Aldine de Gruyter.

Wright, Lory E.

1997 "Ecology or Society? Paleodiet and the Collapse of the Pasión Maya Lowlands", Bones of the Maya. Studies of Ancient Skeletons, S. L. Whittington y D. M. Reed (eds.), pp: 181-195. Washington: Smithsonian Institution Press. 